\title{
NOD-like receptors mediate inflammatory lung injury during plateau hypoxia exposure
}

\author{
Haiyan Wang ${ }^{1}$, Xue Lin ${ }^{1}$ and Xiaoyan $\mathrm{Pu}^{1,2^{*}}$
}

\begin{abstract}
Background: The lung is an important target organ for hypoxia treatment, and hypoxia can induce several diseases in the body.

Methods: We performed transcriptome sequencing for the lungs of rats exposed to plateau hypoxia at 0 day and 28 days. Sequencing libraries were constructed, and enrichment analysis of the differentially expressed genes (DEGs) was implemented using the Gene Ontology (GO) and Kyoto Encyclopedia of Genes and Genomes (KEGG). Subsequently, experimental validation was executed by quantitative real-time PCR (qRT-PCR) and western blot.

Results: The results showed that the nucleotide-binding oligomerization domain (NOD)-like receptor (NLR) signaling pathway that was involved in immunity may play a crucial function in lung injury caused by plateau hypoxia. And the expressions of NOD1, NOD2, IL-1 $\beta, T N F-a, I L-6$, and $I L-18$ were higher at 28 days of exposure to plateau hypoxia than that at 0 day. Similarly, CARD9, MYD88, p38 MAPK, and NF-KB p65, which are related to the NF-KB and MAPK signaling pathways, also demonstrated increased expression at 28 days exposure to plateau hypoxia than at 0 day.

Conclusions: Our study suggested that the NFKBp65 and p38 MAPK signaling pathways may be activated in the lungs of rats during plateau hypoxia. Upregulated expression of NFKBp65 and p38 MAPK can promote the transcription of downstream inflammatory factors, thereby aggravating the occurrence and development of lung tissue remodeling.
\end{abstract}

Keywords: Plateau hypoxia, Lung, Inflammatory, Transcriptome sequence, NOD-like receptors

\section{Introduction}

About $2 \%$ of the world's total population lives in highaltitude areas (generally $\geq 2500 \mathrm{~m}$ ), and in China alone, areas above $3000 \mathrm{~m}$ account for $25 \%$ of the country's land [1]. Moreover, with the continuous development of tourism, the economy, and national defense, increasing numbers of people will enter high-altitude areas to live and work [2]. The plateau is an ecological environment

\footnotetext{
* Correspondence: 878794913@qq.com

'College of Medicine, Qinghai University, Xining 810001, Qinghai Province, China

${ }^{2}$ Qinghai Normal University, Xining 810007, Qinghai Province, China
}

which possesses some special features, such as low oxygen, high radiation, and low pressure [3]. When humans access the plateau environment from the plains, their organs and tissues undergo physiological hypoxia. High altitude actuates the body to decrease partial pressure and oxygen saturation of the arterial blood, eventually leading to tissue hypoxia [4]. On the other hand, hypoxia can cause high-altitude pulmonary edema (HAPE) [5], high-altitude cerebral edema (HACE) [6], polycythemia, and cardiovascular diseases [7]. As the lung is the most sensitive organ to oxygen, hypoxia can cause lung damage. Especially concerning is the risk that acute high-

(c) The Author(s). 2020 Open Access This article is licensed under a Creative Commons Attribution 4.0 International License, which permits use, sharing, adaptation, distribution and reproduction in any medium or format, as long as you give appropriate credit to the original author(s) and the source, provide a link to the Creative Commons licence, and indicate if changes were made. The images or other third party material in this article are included in the article's Creative Commons licence, unless indicated otherwise in a credit line to the material. If material is not included in the article's Creative Commons licence and your intended use is not permitted by statutory regulation or exceeds the permitted use, you will need to obtain permission directly from the copyright holder. To view a copy of this licence, visit http://creativecommons.org/licenses/by/4.0/ The Creative Commons Public Domain Dedication waiver (http://creativecommons.org/publicdomain/zero/1.0/) applies to the data made available in this article, unless otherwise stated in a credit line to the data. 
altitude hypoxia or mild altitude reactions can result in HAPE [5].

As with all pattern-recognition receptors (PRRs), NLRs are located intracellularly $[8,9]$ and recognize pathogenassociated molecular patterns (PAMPs) [10] to defend the host from invading bacteria, viruses, and other pathogens. There are 22 NLRs in humans and at least 33 NLRs in mice. The majority of NLRs comprise a variable $\mathrm{N}$-terminal effector domain, a central NOD, and a Cterminal leucine-rich repeat (LRR) domain [8]. The NLR family includes five subfamilies according to the type of effector domain, which is either a caspase recruitment domain (CARD), a baculoviral inhibitor of apoptosis protein repeat (BIR) domain, or a pyrin domain (PYD) [11]. Among the five subfamilies, the NLRC subfamily is comprised of the CARD-containing molecules NOD1, NOD2, and NLRC3-5 [11]. The earliest discovered NLRs [12, 13], NOD1, and NOD2 are both expressed in the lungs [14-20]. After NOD1 and NOD2 recognize the PAMPs, the nuclear factor (NF) $-\mathrm{kB}$ and MAP kinase (MAPK) signaling pathways are activated through Rip2 kinase. Subsequently, pro-inflammatory cytokines, such as TNF $\alpha$, IL-1 $\beta$, IL-6, and IL-18, chemokines, and antimicrobial peptides are activated and secreted to blossom an effective immune response. Typically, severe inflammatory responses are found in HAPE patients [21]. Therefore, we speculate that the NOD-like receptor signaling pathway may be tightly involved with the occurrence and development of HAPE.

Our previous results revealed increased alveolar compensation or increased alveolar capillaries by Hematoxylin and Eosin (HE) staining when the lung had been exposed to plateau hypoxia for 28 days (unpublished data). However, the molecular mechanism of damage to the lungs of rats exposed to plateau hypoxia is still vague. In this present study, transcriptome sequencing methods were employed to research the effect of plateau hypoxia on rat lungs. Additionally, based on the transcriptome analysis results, we verified the expression of certain genes and proteins related to the NF$\mathrm{KB}$ and MAPK signaling pathways by the quantitative real-time PCR (qRT-PCR) and western blot, respectively. These data present a new avenue to study the molecular regulation mechanism of plateau hypoxia on the lungs.

\section{Materials and methods}

\section{Animals}

Twelve specific-pathogen-free (SPF) rats, weighing $200 \pm$ $20 \mathrm{~g}$, were bought from the Medical Laboratory of Xi'an Jiaotong University, experimental animal license number: SCXK (shan) 2018-001. The temperature of the rearing room was $25 \pm 2^{\circ} \mathrm{C}$, and the relative humidity was 40 to $60 \%$. All rats were fed a standard diet and had free drinking water. Twelve rats were randomly divided into two groups of six rats each:28-day group and 0 day group. In the 28-day group: These six rats were raised in Maduo County, Qinghai Province, at an altitude of 4200 $\mathrm{m}$ for 28 days to collect the lung tissues. In the 0-day group: These six rats were housed at the Medical Laboratory of Xi'an Jiaotong University at an altitude of $500 \mathrm{~m}$ and then sacrificed at day 0 in this study. Therein, three rats of each group were used for RNA extracts, which was divided into two parts for mRNA-seq and qRT-PCR analysis, respectively. Another three rats were used for western blot analysis. All animals were used in accordance with all animal welfare laws, and the study was approved by the Ethical Committee of Qinghai University.

\section{RNA-seq library preparation and Illumina sequencing}

The total RNA from the lungs of rats exposed to plateau hypoxia at 0 days and 28 days was extracted using an Animal Total RNA Isolation Kit (RE-03014, FOREGENE, Chengdu, China) in accordance with the manufacturer's instructions. The purity and integrity of the obtained RNA were also quantified by an Agilent BioAnalyzer 2100. Each RNA sample was both used for transcriptome sequencing and experimental validation through qRT-PCR. The RNA sample from one individual at 0 day or 28 days was collected to generate one replicate sample. Equal amounts of RNA from the 0-day and 28-day samples were employed for the construction of mRNA-Seq libraries. An Illumina TruSeq RNA Sample Prep Kit was used to construct the mRNA-Seq libraries at Beijing Novogene Zhiyuan Technology Co., Ltd. (Beijing, China). In brief, the poly-T oligo-attached magnetic beads were employed to purify mRNA from total RNA. Subsequently, the purified mRNA was fragmented and reversely transcribed into cDNA. Then, connect the adaptor to the cDNA molecule and amplify the fragment by PCR. The Illumina HiSeq 2500 sequencing platform was used to execute the sequencing in paired-end reads $(2 \times 101 \mathrm{bp})$. The FastQC method was used to evaluate the quality of data from mRNA sequencing (http://www.bioinformatics.babraham.ac.uk/projects/fastqc/).

\section{Bioinformatics analysis of mRNA-seq data}

The mRNA-Seq reads generated by the Illumina HiSeq platform. Subsequently, the adaptor sequences and the low-quality $(<20)$ bases at the $5^{\prime}$ and $3^{\prime}$ ends were removed by Trimmomatic (v0.30) [22]. The clean reads longer than $70 \mathrm{bp}$ were employed for further experiment. The reads were mapped to the Rattus norvegicus genome (Ensembl RGSC3.4) using TopHat v1.4.1 [23] with default parameters $(-r 400-p$ 8) after preprocessing of mRNA-Seq data. The gene abundances were quantified using the RSEM (https://www.biostat.wisc.edu/ cdewey/). 
The readings were normalized via DEseq2 v. 1.24 .0 (http://bioconductor.org/packages/stats/bioc/DESeq2/) [24] and was used for differential expression analysis of read counts. The following criteria are used here: Default parameters: Benjamini \& Hochberg $(\mathrm{BH}) p$ adjust $<0.05 \&\left|\log _{2} \mathrm{FC}\right| \geq 1$ after multiple comparisons. The resulting $p$ values were adjusted through $\mathrm{BH}$ false discovery rate (FDR) algorithm. Differential expression of genes was significant only when the FDR values were $<0.05$ (controlling the expected FDR to no more than 5\%) and the $\log _{2}$ FoldChange (FC) was $\geq 1$. This tool employs a negative binomial distribution model to detect for differential gene expression. Ami GO with the default parameters were employed to acquire gene ontology terms of each gene and analyze GO functional enrichment via hypergeometric tests with FDR correction to gain an adjusted $p$ value between particular test gene groups and the whole annotation data set, severally. The DEGs in Kyoto Encyclopedia of Genes and Genomes (KEGG) pathway were analyzed using Cytoscape with the ClueGO plugin $[25,26]$.

\section{CDNA synthesis and real-time PCR analysis of gene expression}

cDNA synthesis was performed using a PrimeScript RT reagent kit (RR047A, Takara, Japan) following the manufacturer's instructions. qRT-PCR was performed using the A PIKORed 96 (ThermoFisher, USA) with primers (listed in Table 1) and using the TB Green TM Premix Ex TaqTM II (Tli RNaseH Plus) (Takara, RR820A) as described previously [27]. The expression of each gene was first normalized to that of $\beta$-actin and was presented as a fold change by calculating the average expression level of each of the three samples divided by that of the controls at the same time point.

\section{SDS-PAGE and western blot analysis}

The total protein was extracted from rat lung samples, and the protein concentration was quantified using the BCA protein quantification kit. SDS-PAGE and western blot analyses were executed as the previous study [28].
In brief, the proteins were separated by $12 \%$ SDS-PAGE and then were electrically transferred to a PVDF membrane. The PVDF membrane was incubated with the primary antibody (the primary antibody and the corresponding dilution concentration are listed in Table 2) at $4{ }^{\circ} \mathrm{C}$ overnight after pre-blocking with TBST (containing $5 \%$ skim milk (Anchor, New Zealand)) for $2 \mathrm{~h}$ at room temperature. The membrane was incubated with goat-antirabbit IgG (H\&L)-HRP or goat-anti-mouse IgG (H\&L)HRP (Abcam, Cambridge, UK) diluted 1:5000 in TBST (containing 5\% skim milk) for $2-3 \mathrm{~h}$ at room temperature after three washes with TBST. The reaction was visualized using an ECL Luminescence Kit (affinity, KF001) for $1 \mathrm{~min}$. Scan analysis was performed using a gel image analysis imaging system, and the results were expressed as the relative expression of the target protein. The relative expression of the target protein $=$ integrated optical density value (IOD) of the target protein/integrated optical density value (IOD) of the internal reference.

\section{Statistical analysis}

The unpaired $t$ test was used to examine the significance of the difference of mean values between two groups using the SPSS 19.0 package (SPSS Inc. Chicago, IL, USA). All results were recorded as the means \pm SE, and the differences were considered statistically nonsignificant, significant, or extremely significant when $p \geq$ $0.05, p<0.05$, and $p<0.01$, respectively.

\section{Results}

\section{Characterization of the transcriptome sequence}

To explore the molecular mechanism of lung damage after exposure to plateau hypoxia, three replicates of the samples at 0 day or 28 days were sequenced, and the transcriptome libraries were constructed. A total of $42.46 \mathrm{M}$ and $58.20 \mathrm{M}$ raw reads were generated for the 0 -day and 28-day samples on average, respectively. Likewise, after the low-quality reads and adapter sequences were filtered out of the raw data, the clean reads were $41.58 \mathrm{M}$ and $56.50 \mathrm{M}$ on average for the 0-day and 28day groups, respectively. The clean read ratio values

Table 1 Primers used in this study

\begin{tabular}{|c|c|c|}
\hline Primer name & Forward primer $\left(5^{\prime}-3^{\prime}\right)$ & Reverse primer $\left(5^{\prime}-3^{\prime}\right)$ \\
\hline$\beta$-actin & GAAGATCAAGATCATTGCTCC & TACTCCTGCTTGCTTGCGATCCA \\
\hline $\mathrm{IL}-1$ & ATCCTCTCCAGTCAGGCTTCCTTGTG & AGCTCTTGTCGAGATGCTGCTGTGA \\
\hline$\| \mathrm{L}-2$ & TGTTGCTGGACTTACAGGTGCTCCT & CCACCACAGTTGCTGGCTCATCATC \\
\hline IL-6 & ACAGAGGATACCACCCACAACAGACC & CGGAACTCCAGAAGACCAGAGCAGAT \\
\hline $\mathbb{I L}-18$ & TGCCTGATATCGACCGAACAGCCAAC & ACAGATAGGGTCACAGCCAGTCCTCT \\
\hline NOD1 & CTCAAAGGAGGACCTGCTGCTGGA & GAAGACAGTCTCGCCATGCTCGTTGA \\
\hline NOD2 & GGCAGCACAGGTGGACTCTGAGGATA & GCAGCAGCCTTAGCAGCAGTGAGTT \\
\hline TNT-a & CCAGCAGGAGGGAGAACAGCAACT & CCGCCACGAGCAGGAATGAGAAGAG \\
\hline
\end{tabular}


Table 2 The primary antibody and the corresponding dilution concentration

\begin{tabular}{llll}
\hline Reagent or resource & Source & Identifier & Dilution concentration \\
\hline Rabbit polyclonal anti-MYD88 & Abcam & Cat\# ab2064 & $1: 1000$ \\
Rabbit polyclonal anti-P-P65 & Abcam & Cat\# ab86299 & $1: 1000$ \\
Mouse polyclonal anti-CARD9 & Abcam & Cat\# ab169623 & $1: 1000$ \\
Mouse polyclonal anti-CD86 & Abcam & Cat\# ab220188 & $1: 1000$ \\
Rabbit polyclonal anti-P-P38 & Abcam & Cat\# ab4822 & $1: 1000$ \\
Rabbit polyclonal anti-B-actin & Abcam & Cat\# ab8227 & $1: 2000$ \\
Goat Anti-Mouse lgG H\&L & Abcam & Cat\# ab6721 & $1: 5000$ \\
Goat Anti-Rabbit lgG H\&L & Abcam & & $1: 5000$ \\
\hline
\end{tabular}

were $97.93 \%$ and $97.09 \%$, the Q30\% values of the clean reads were $93.25 \%$ and $93.14 \%$, and the $\mathrm{GC}$ values were $48.86 \%$ and $50.35 \%$ at 0 day and 28 days on average, respectively (Table 3 ). The uniquely mapped clean reads were utilized in gene expression analysis based on FPKM. After exposure to plateau hypoxia for 28 days, 3474 downregulated and 3278 upregulated genes were present compared to gene expression at 0 day (Fig. 1). The top 100 upregulated and downregulated DEGs $\left(\left|\log _{2} \mathrm{FC}\right| \geq 1\right.$ and FDR $\left.<0.05\right)$ were listed in supplementary files Tables S1 and S2.

GO enrichment analysis of differentially expressed genes after plateau hypoxia exposure at $\mathbf{0}$ day and $\mathbf{2 8}$ days

To further explore the molecular mechanism of lung injury after exposure to plateau hypoxia, DEGs were subjected to GO enrichment analysis relay for the following three categories: biological processes, cellular components, and molecular function (Fig. 2). The results showed that most genes in all three aforementioned categories were involved in the immune process, such as the activation of the immune response and the immune response-regulating signaling pathway at the biological process level, immunoglobulin complex circulating at the cellular components level, and immunoglobulin receptor binding and antigen binding at the molecular function level. In brief, GO enrichment analysis suggested that immunity may play an important role in lung injuries caused by plateau hypoxia.

\section{KEGG enrichment analysis of differentially expressed genes after plateau hypoxia exposure at 0 day and 28 days}

To acknowledge the role of immunity in lung injuries caused by plateau hypoxia, KEGG enrichment analysis was carried out on top 20 most enriched pathways of DEGs (Fig. 3). Enriched KEGG pathways from the DEGs are listed in supplementary files Table S3. Among the many enriched signaling pathways, the NOD-like receptor signaling pathway involved in immunity was significantly upregulated, which suggested that the NOD-like receptor signaling pathway may play a crucial function in lung injury caused by plateau hypoxia. The KEGG enrichment map plots of NOD-like receptor signaling pathway were shown in Fig. 4, and 46 upregulated DEGs and 11 downregulated DEGs were involved in NOD-like receptor signaling pathway (listed in supplementary files Table S4).

\section{Gene expression of the lung exposed to plateau hypoxia at 0 day and 28 days}

Based on the transcriptome sequence analysis, the mRNA expression of six genes related to the NOD-like

Table 3 Summary of the reads and mapping results

\begin{tabular}{lllllllll}
\hline Sample & $\mathbf{0 - 1}$ days & $\mathbf{0 - 2}$ days & $\mathbf{0 - 3}$ days & $\mathbf{0 - d a y}$ mean & $\mathbf{2 8 - 1}$ day & $\mathbf{2 8 - 2}$ days & $\mathbf{2 8 - 3}$ days & 28-day mean \\
\hline Total raw reads (M) & 39.91 & 44.84 & 42.63 & 42.46 & 61.40 & 57.87 & 55.32 & 58.20 \\
Total clean reads (M) & 39.22 & 43.79 & 41.72 & 41.58 & 59.69 & 56.06 & 53.75 & 56.50 \\
Total clean bases (G) & 5.88 & 6.57 & 6.26 & 6.24 & 8.95 & 8.41 & 8.06 & 8.47 \\
Clean reads Q20 (\%) & 97.91 & 97.75 & 96.72 & 97.46 & 97.35 & 97.79 & 97.31 & 97.48 \\
Clean reads Q30 (\%) & 94.19 & 93.86 & 91.69 & 93.25 & 92.82 & 93.82 & 92.77 & 93.14 \\
Clean reads ratio (\%) & 98.28 & 97.65 & 97.85 & 97.93 & 97.22 & 96.88 & 97.16 & 97.09 \\
Total mapping (\%) & 93.10 & 92.94 & 92.37 & 92.80 & 95.05 & 94.34 & 94.7 & 94.70 \\
Multiple mapping (\%) & 4.6 & 4.91 & 4.51 & 4.67 & 5.6 & 5.98 & 5.56 & 5.71 \\
Unique mapping (\%) & 88.51 & 88.04 & 87.86 & 88.14 & 89.44 & 88.37 & 89.14 & 88.98 \\
GC (\%) & 48.66 & 49.18 & 48.75 & 48.86 & 50.36 & 50.54 & 50.14 & 50.35 \\
\hline
\end{tabular}




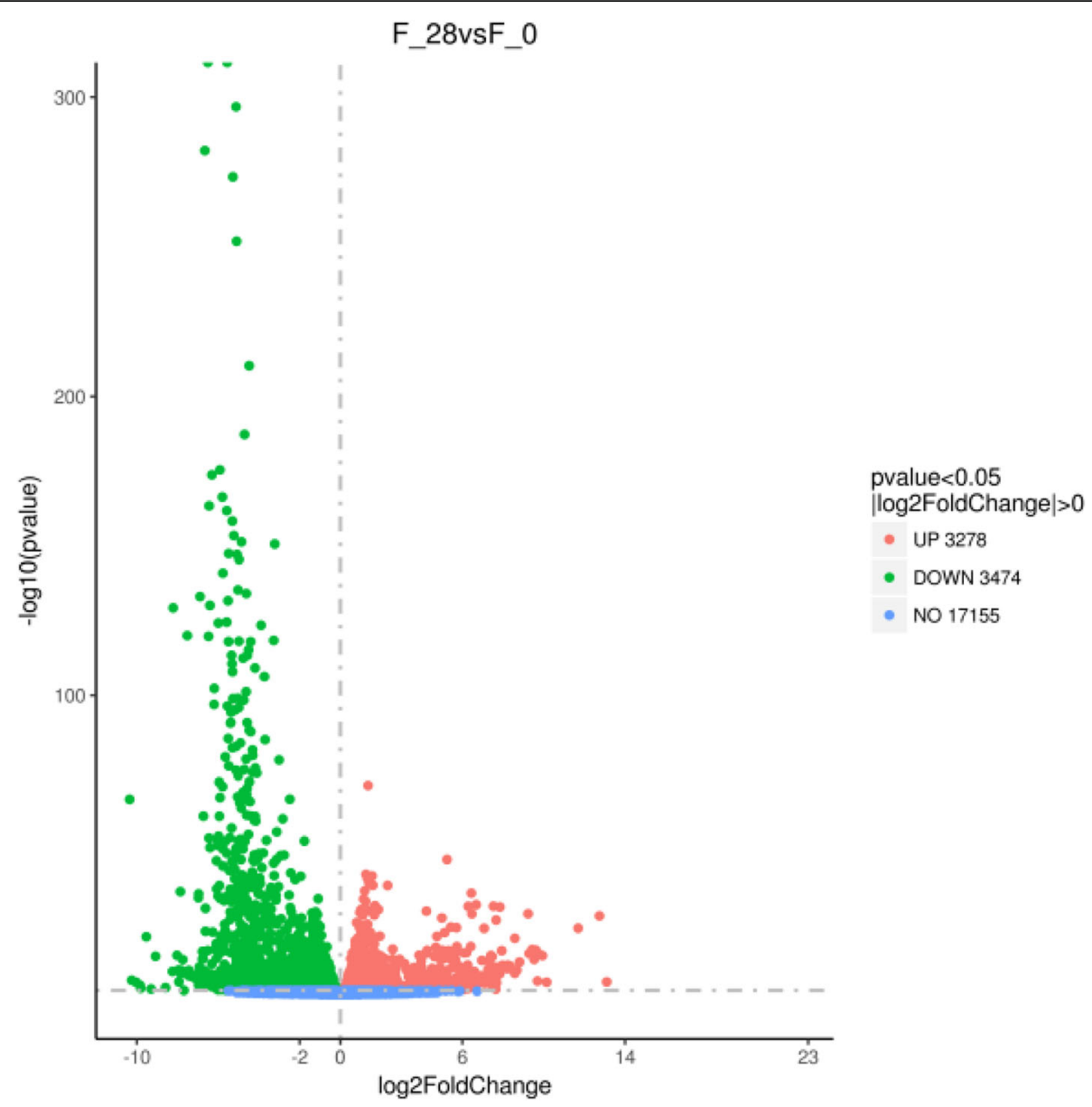

Fig. 1 A Volcano plot of genes identified between 0 day and 28 days

receptor signaling pathway was detected using qRTPCR. As both NOD1 and NOD2 were expressed in the lungs of rats, we detected the expression of NOD1 and NOD2 after exposure to plateau hypoxia at 0 day and 28 days. We found that both NOD1 and NOD2 expression were significantly higher at a 28-day exposure than that at 0 day (Fig. 5a, b).

Similarly, some inflammatory factors were expressed after plateau hypoxia exposure. $I L-1 \beta$ and $T N F-\alpha$ expression remained higher at 28 days than at 0 day of plateau hypoxia exposure (Fig. 4c, f). Importantly, the expression of $I L-6$ and $I L-18$ was significantly higher at 28 days than that at 0 day of plateau hypoxia exposure (Fig. $5 \mathrm{~d}, \mathrm{e}$ ).

\section{Protein expression of the lung at 0 day and 28 days of plateau hypoxia exposure}

Furthermore, the expression of proteins associated in the NOD-like receptor signaling pathway was examined using western blot. As NLRs can activate the NF- $k B$ and MAPK signaling pathways, we detected the expression of some key proteins related to these two pathways. Upon recognition of PAMPs, NOD recruits adaptor proteins MyD88 and CARD9 to activate the NF- $\mathrm{KB}$ and MAPK pathways. Thus, we detected the protein expression of MyD88 and CARD9. The results showed that the expression of CARD9 and MYD88 was significantly higher at 28 days than that at 0 day of plateau hypoxia exposure. Since activation of the p38 MAPK signaling pathway can further directly activating the NF$\kappa \mathrm{B}$ p65 pathway, we found that the expression of phosphorylated p38 MAPK and NF-кBp65 was extremely significantly higher at 28 days than that at 0 day (Figs. 6 and 7). In brief, these results indicated that the NF- $\mathrm{BB}$ and MAPK pathways are activated when the rats are exposed to plateau hypoxia.

\section{Discussion}

The lung is the most sensitive organ to hypoxia. When the partial pressure of oxygen in the alveoli decreases to a certain threshold, rapid pulmonary artery contraction may occur, and corresponding changes in the bronchi and alveolar cells in the lung will also occur. In this present study, the transcriptome analysis obtained from GO and KEGG enrichment analysis of the DEGs showed 


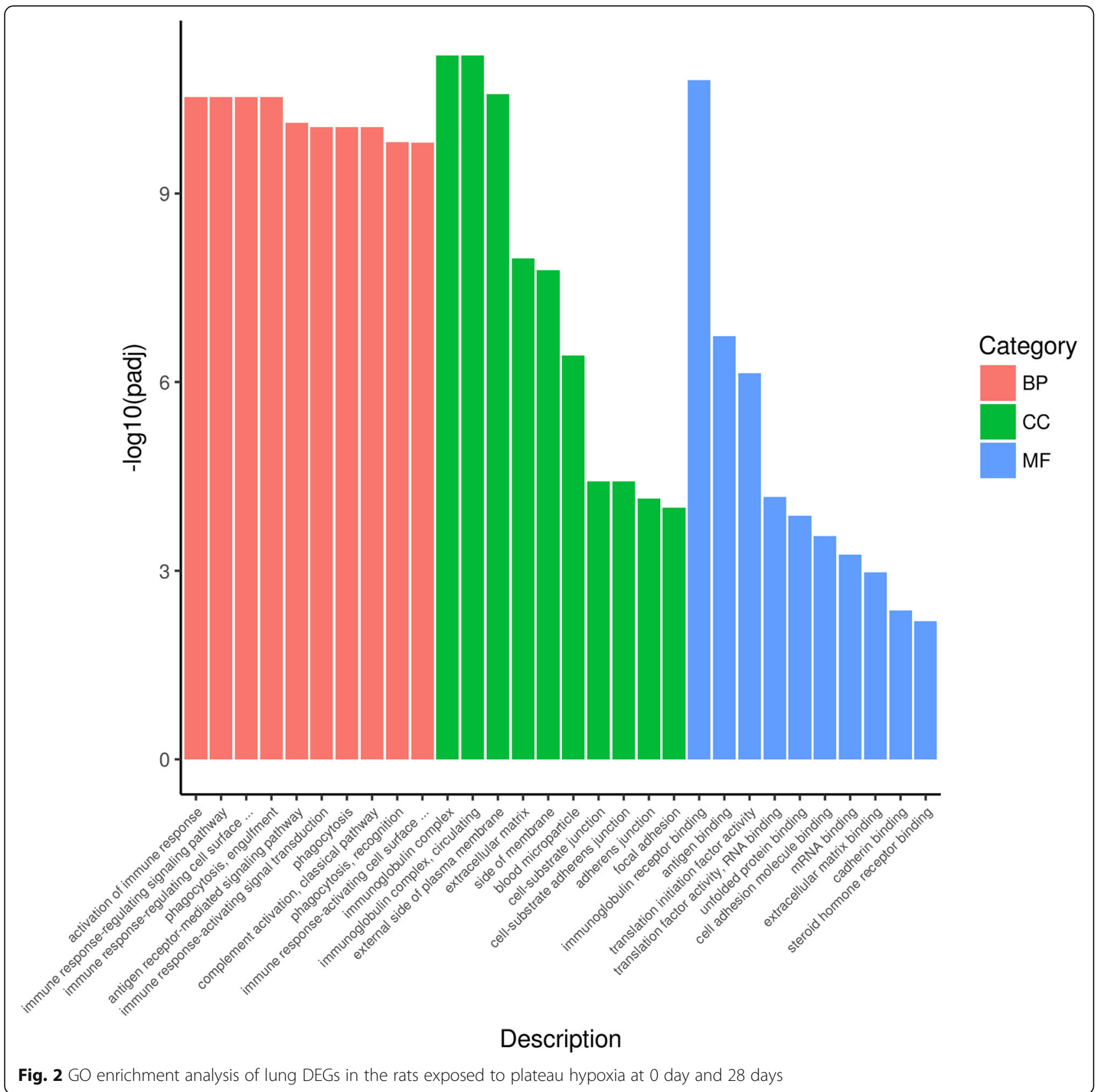

that the NOD-like receptor signaling pathway involved in immunity may play an important role in lung injury caused by plateau hypoxia. Further experimental validation suggested that the NFkBp65 and p38 MAPK signaling pathways were activated in the lungs of rats during plateau hypoxia. Upregulated expression of NF$\kappa B p 65$ and p38 MAPK can promote the transcription of downstream inflammatory factors, thereby aggravating the occurrence and development of lung tissue remodeling.

With the increasing progress of transcriptomic technology, lung transcriptome sequencing has been studied extensively. The transcriptome sequence of the lung in mice infected with influenza A virus [29] and that of the PRRSV infection in porcine lungs [30] have been analyzed. Meanwhile, other research focuses on transcriptome analysis of sick or cancerous lungs [31-33]. Additionally, the transcriptome analysis of lung after treated with adenocarcinoma drugs has also gained attention [34, 35]. After mice were infected with influenza A virus, the expression of virus-induced chemokines, pro-inflammatory cytokines, adhesion molecules, and inflammatory cells and inflammatory enzymes, antibodies and complement activation were upregulated. 


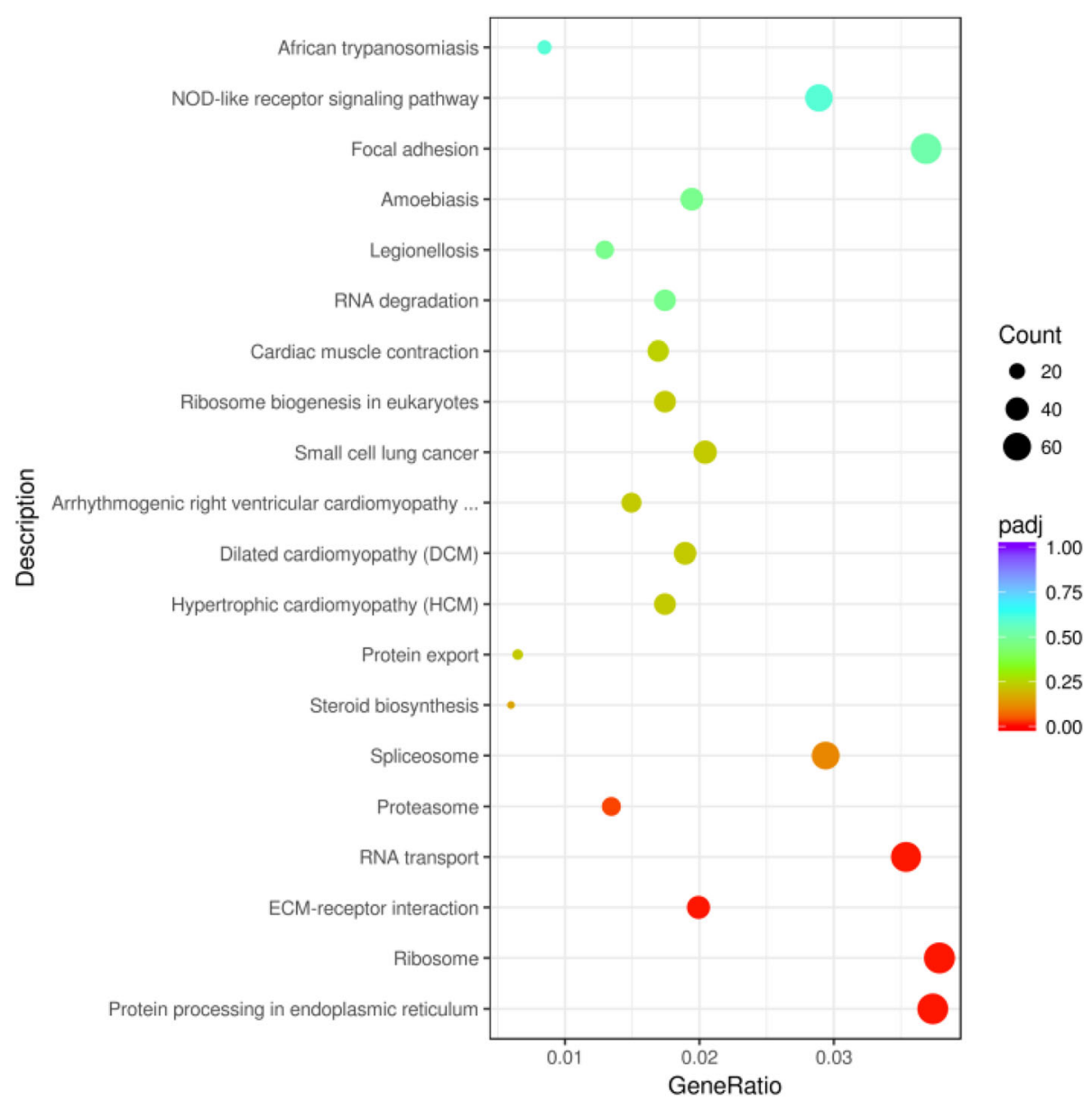

Fig. 3 KEGG enrichment analysis of lung DEGs in the rats exposed to plateau hypoxia at 0 day and 28 days

Meanwhile, the transcriptome analysis of lung after treated with adenocarcinoma drugs revealed that majority of the identified genes were enriched in the PI3K/ AKT, actin cytoskeleton regulation, mitogen-activated protein kinase, and focal adhesion pathways [34]. In the present study, we executed the transcriptome analysis as in a previous study of transcriptome analysis of the yak distributed in the Qinghai-Tibetan Plateau that the biological process analysis showed that those involved in immunological cells accounted for the highest ratio [36]. The experimental validation with qRT-PCR and western blot was utilized to obtain results suggesting that the NOD-like receptors mediate inflammatory lung injury after plateau hypoxia exposure.

Generally, NOD1 and NOD2 were both found in the lung. NOD1 has been found in endothelial cells, human airway smooth muscle cells, lung epithelial cells, and different types of leukocytes [14-17], while NOD2 is expressed in neutrophils, bronchial epithelial cells, and alveolar macrophages [18-20]. Thus, we analyzed the expression of NOD1 and NOD2 after the rats were exposed to plateau hypoxia. After PAMPs recognize NOD genes both the NF- $\mathrm{kB}$ and MAPK pathways were activated. Subsequently, certain inflammatory cytokines and chemokines such as IL-1 $\beta$, TNF- $\alpha$, IL-6, and IL-18, which are pivotal for stimulation and recruitment of additional effector cells, were secreted to develop an effective immune response [15, 37-39]. Hence, we determined the expression of these genes above. As major regulators of inflammation and immunity, IL- $1 \beta$, TNF- $\alpha$, IL-6, and IL-18 play important roles in initiating inflammatory reactions $[40,41]$. The results suggested that the expression levels of the examined genes, including NOD1, NOD2, IL-1 $, T N F-\alpha, I L-6$, and $I L-18$, were all increased after exposure to plateau hypoxia. These results indicated that plateau hypoxia may cause an inflammatory reaction in the lungs of rats.

Additionally, upon recognition of their respective PAMPs, NOD recruits adaptor proteins such as MyD88 and CARD9 to activate the NF- $\mathrm{kB}$ and MAPK pathways [42]. It has been shown that p38 MAPK participates in the activation of NF- $\mathrm{kB}$. Activation of the p38 MAPK signaling pathway can promote I $\mathrm{B} \alpha$ double phosphorylation and degradation, thereby directly activating the 

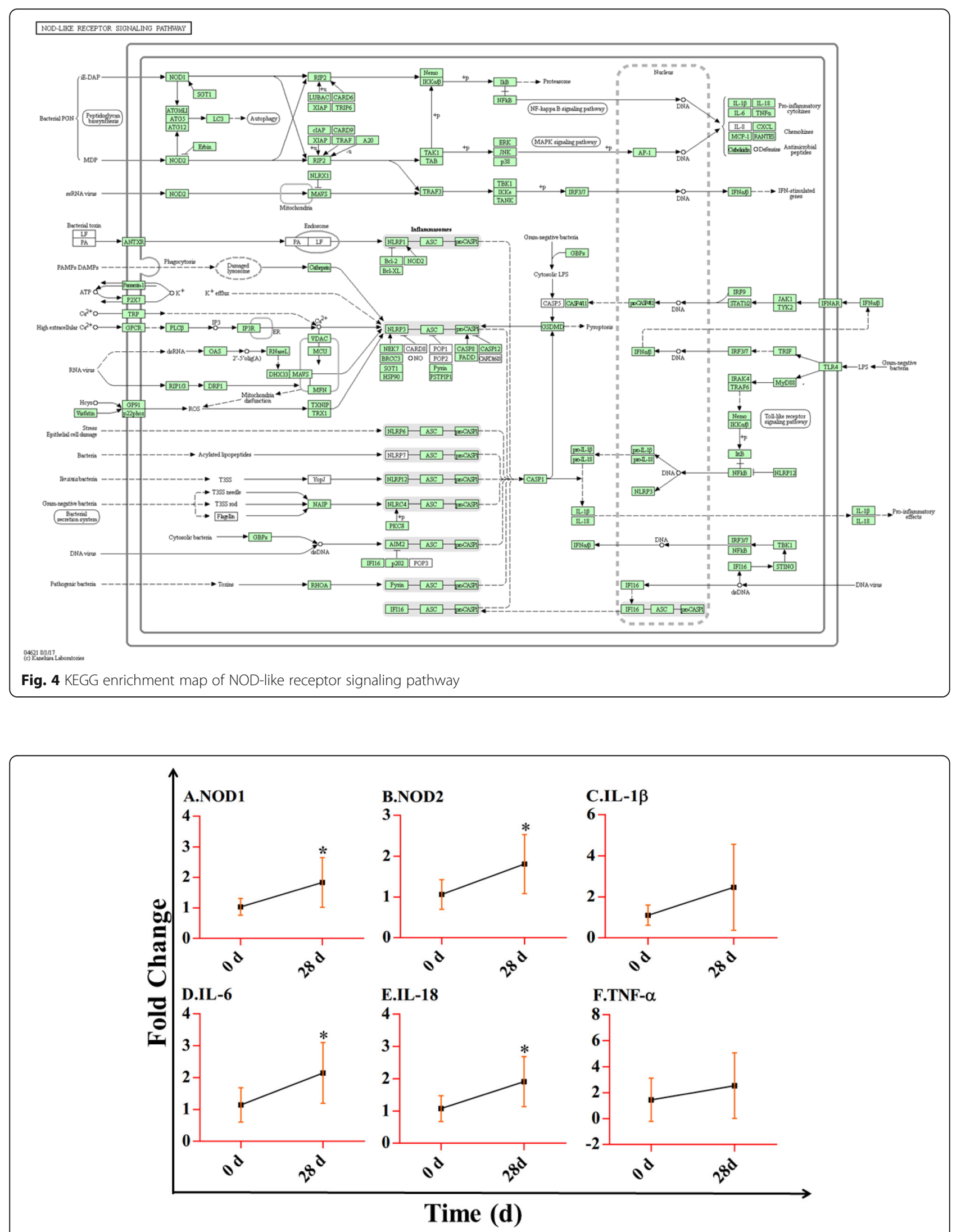

Fig. 5 The expression of NOD1, NOD2, IL-1 $\beta, T N F-a, I L-6$, and $I L-18$ in the lungs of rats exposed to plateau hypoxia at 0 day and 28 days 


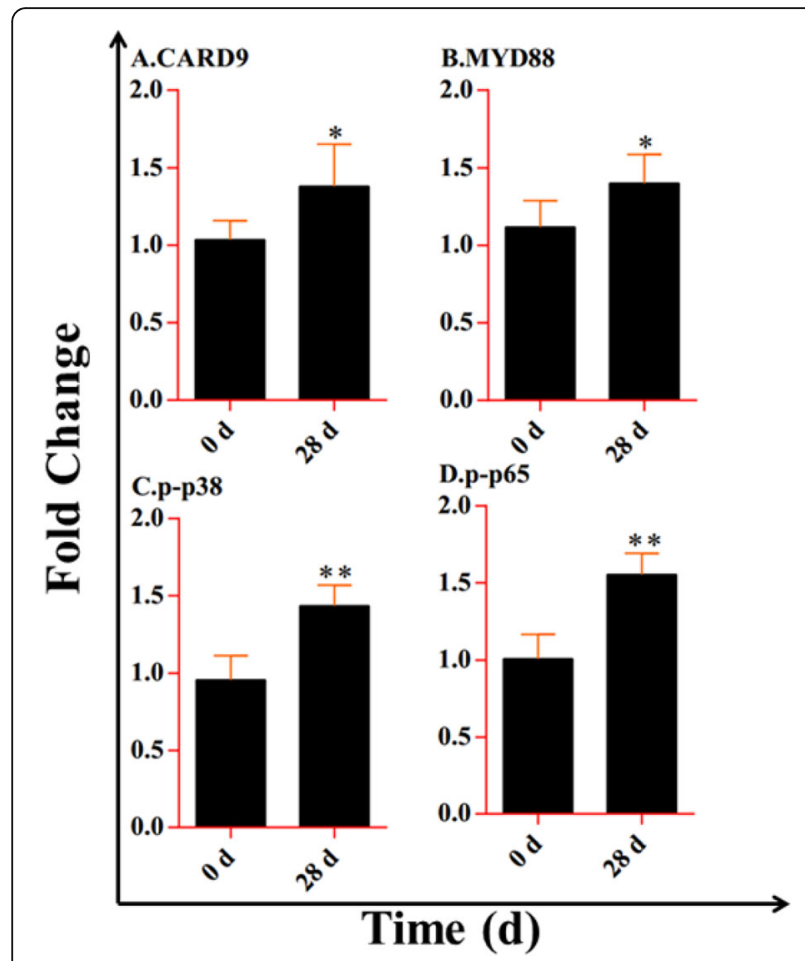

Fig. 6 The expression of CARD9, MYD88, p-p38, and p-p65 in the lungs of rats exposed to plateau hypoxia at 0 day and 28 day

NF-kB p65 pathway, suggesting that p38 MAPK may participate in the pathological damage of lung tissue by activating NF- $\kappa B$ p65 [43]. Our results also revealed that the expression levels of the examined proteins, including CARD9, MyD88, p38 MAPK, and NF- $k B$ p65, were all increased after exposure to plateau hypoxia, indicating that the NF- $\mathrm{kB}$ and MAPK pathways are activated when the rats are exposed to plateau hypoxia.

In summary, the present study demonstrated that NOD-like receptors can mediate inflammation while the NFkBp65 and p38 MAPK signaling pathways may be activated in the lungs of rats during plateau hypoxia.

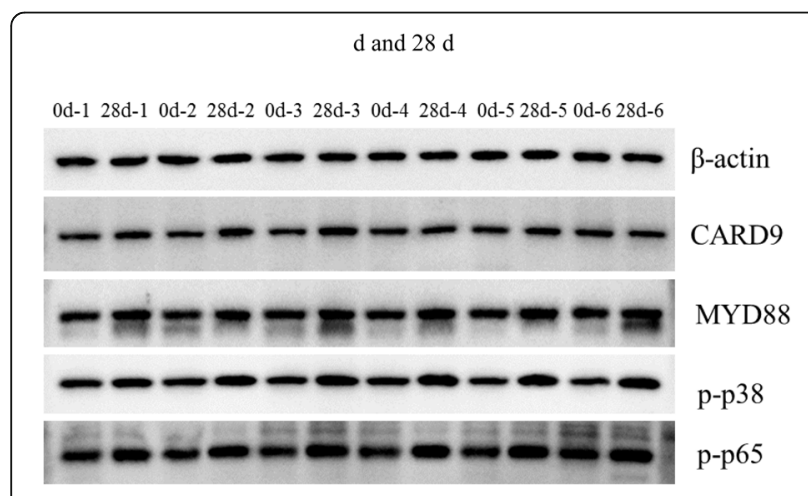

Fig. 7 Western blot analysis of CARD9, MYD88, p-p38, and p-p65 in the lungs of rats exposed to plateau hypoxia at 0 day and 28 days
Upregulated expression of NFkBp65 and p38 MAPK can promote the transcription of downstream inflammatory factors, thereby aggravating the occurrence and development of lung tissue remodeling. Our research provides a novel insight into the molecular mechanism of lung injury in rats after plateau hypoxia exposure.

\section{Supplementary information}

Supplementary information accompanies this paper at https://doi.org/10. 1186/s40101-020-00242-W

Additional file 1: Table S1. List of top 100 upregulated DEGs expressed between $0 \mathrm{~d}$ and $28 \mathrm{~d}$.

Additional file 2: Table S2. List of top 100 downregulated DEGs expressed between $0 \mathrm{~d}$ and $28 \mathrm{~d}$.

Additional file 3: Table S3. Enriched KEGG pathways from DEGs between $0 \mathrm{~d}$ and $28 \mathrm{~d}$

Additional file 4: Table S4. The detail information of DEGs derived from NOD-like receptor signaling pathway.

\section{Abbreviations}

DEGs: The differentially expressed genes; GO: Gene Ontology; KEGG: Kyoto Encyclopedia of Genes and Genomes; qRT-PCR: Real-time PCR:

NLR: Nucleotide-binding oligomerization domain-like receptor; HAPE: Highaltitude pulmonary edema; HACE: High-altitude cerebral edema;

PRRs: Pattern-recognition receptors; PAMPs: Pathogen-associated molecular patterns; CARD: Caspase recruitment domain; NOD: Nucleotide-binding oligomerization domain; CARD: Caspase recruitment domain; IOD: Integrated optical density value

\section{Acknowledgements}

We thank LetPub (www.letpub.com) for its linguistic assistance during the preparation of this manuscript.

\section{Authors' contributions}

Haiyanwang contributed to the idea of the study and played an important role in interpreting the results. Xue Lin contributed significantly to analysis and manuscript preparation; Xiaoyan Pu helped perform the analysis with constructive discussion. All authors read and approved the final manuscript.

\section{Funding}

This research was supported by the Second Tibetan Plateau Scientific Expedition and Research Program, Grant No. 2019QZKK0606, Project 31660254 of the Chinese National Nature Science Foundation, Project 2017ZJ-Y13 of the Key Laboratory of Medicinal Animal and Plant resources of the Qinghai-Tibetan Plateau, Projects 2019-ZJ-7042 of the Department of Science and Technology of Qinghai Province, and Projects 201910743003 of National Students' platform for innovation and entrepreneurship training Program.

Availability of data and materials

The datasets used and/or analyzed during the current study are available from the corresponding author upon reasonable request.

Ethics approval and consent to participate

The animals involved in the study were handled in accordance with the National Regulations on the Administration of Laboratory Animals (GB149232010). The animal experiment scheme was approved and examined by the Ethics Committee of School of Medicine of Qinghai University.

Consent for publication

Not applicable.

Competing interests

The authors declare that there is no conflict of interest. 
Received: 11 June 2020 Accepted: 28 September 2020

Published online: 07 October 2020

\section{References}

1. Singh LC. High altitude dermatology. Indian J Dermatol. 2017;62(1):59-65.

2. Zafren K. Prevention of high altitude illness. Travel Med Infect Dis. 2014; 12(1):29-39.

3. West JB. Recent advances in high altitude medicine and biology. High Alt Med Biol. 2015:16(2):73.

4. Horscroft JA, et al. Metabolic basis to Sherpa altitude adaptation. Proc Natl Acad Sci U S A. 2017:114(24):6382-7.

5. Richalet JP, et al. Physiological risk factors for severe high-altitude illness: a prospective cohort study. Am J Respir Crit Care Med. 2012;185(2):192-8.

6. Joyce $\mathrm{KE}$, et al. Advances in the available non-biological pharmacotherapy prevention and treatment of acute mountain sickness and high altitude cerebral and pulmonary oedema. Expert Opin Pharmacother. 2018;19(17): 1891-902.

7. Zheng $C$, et al. Metabolic risk factors and left ventricular diastolic function in middle-aged chinese living in the Tibetan plateau. J Am Heart Assoc. 2019; 8(6):e010454.

8. Chen G, et al. NOD-like receptors: role in innate immunity and inflammatory disease. Annu Rev Pathol. 2009;4:365-98.

9. Magalhaes JG, et al. What is new with Nods? Curr Opin Immunol. 2011; 23(1):29-34

10. Janeway CA Jr. Approaching the asymptote? Evolution and revolution in immunology. Cold Spring Harb Symp Quant Biol. 1989;54(Pt 1):1-13.

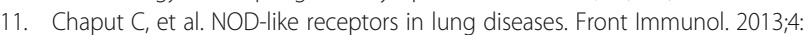
393.

12. Inohara N, et al. Nod1, an Apaf-1-like activator of caspase-9 and nuclear factor-kappaB. J Biol Chem. 1999;274(21):14560-7.

13. Ogura $Y$, et al. Nod2, a Nod1/Apaf-1 family member that is restricted to monocytes and activates NF-?B. J Biol Chem. 2001;276(7):4812-8.

14. Slevogt $\mathrm{H}$, et al. Moraxella catarrhalis is internalized in respiratory epithelial cells by a trigger-like mechanism and initiates a TLR2- and partly NOD1dependent inflammatory immune response. Cell Microbiol. 2007;9(3):694.

15. Opitz B, et al. Listeria monocytogenes activated p38 MAPK and induced IL-8 secretion in a nucleotide-binding oligomerization domain 1-dependent manner in endothelial cells. J Immunol. 2006;176(1):484-90.

16. Barton $\mathrm{L}$, et al. The pattern recognition receptor Nod1 activates CCAAT/ enhancer binding protein beta signalling in lung epithelial cells. Eur Respir J. 2007;30(2):214-22

17. Mansson Kvarnhammar A, et al. Innate immune receptors in human airway smooth muscle cells: activation by TLR1/2, TLR3, TLR4, TLR7 and NOD1 agonists. PLoS One. 2013;8(7):e68701.

18. Opitz B, et al. Nucleotide-binding oligomerization domain proteins are innate immune receptors for internalized Streptococcus pneumoniae. J Biol Chem. 2004;279(35):36426-32.

19. Theivanthiran $B$, et al. NOD2 signaling contributes to host defense in the lungs against Escherichia coli infection. Infect Immun. 2012;80(7):2558-69.

20. Shimada K, et al. The NOD/RIP2 pathway is essential for host defenses against Chlamydophila pneumoniae lung infection. PLoS Pathog. 2009:5(4): e1000379.

21. Bärtsch P. High altitude pulmonary edema. Med Sci Sports Exerc. 1999;31(1 Suppl):S23-7.

22. Bolger AM, Lohse M, Usadel B. Trimmomatic: a flexible trimmer for Illumina sequence data. Bioinformatics. 2014;30(15):2114-20.

23. Trapnell C, Pachter L, Salzberg SL. TopHat: discovering splice junctions with RNA-Seq. Bioinformatics. 2009;25(9):1105-11.

24. Anders S, Huber W. Differential expression analysis for sequence count data. Genome Biol. 2010;11(10):R106.

25. Mao $X$, et al. Automated genome annotation and pathway identification using the KEGG Orthology (KO) as a controlled vocabulary. Bioinformatics. 2005;21(19):3787-93.

26. Young MD, et al. Gene ontology analysis for RNA-seq: accounting for selection bias. Genome Biol. 2010;11(2):R14.

27. Wang $T$, et al. Two copies of the genes encoding the subunits of putative interleukin (IL)-4/IL-13 receptors, IL-4Ra, IL-13Ra1 and IL-13Ra2, have been identified in rainbow trout (Oncorhynchus mykiss) and have complex patterns of expression and modulation. Immunogenetics. 2011;63(4):235-53.
28. Xie $Y$, et al. Potential of recombinant inorganic pyrophosphatase antigen as a new vaccine candidate against Baylisascaris schroederi in mice. Vet Res. 2013;44(1):90

29. Hu J, et al. Deep sequencing of the mouse lung transcriptome reveals distinct long non-coding RNAs expression associated with the high virulence of H5N1 avian influenza virus in mice. Virulence. 2018;9(1):1092111.

30. Xiao $S$, et al. Understanding PRRSV infection in porcine lung based on genome-wide transcriptome response identified by deep sequencing. PLoS One. 2010;5(6):e11377

31. Kim WJ, et al. Comprehensive analysis of transcriptome sequencing data in the lung tissues of COPD subjects. Int J Genomics. 2015;2015:206937.

32. Yang $L L$, et al. Integrative analyses of lung squamous cell carcinoma in ten Chinese patients with transcriptome sequencing. J Genet Genomics. 2015; 42(10):579-87.

33. Ma J, et al. Characterization of microRNA transcriptome in lung cancer by next-generation deep sequencing. Mol Oncol. 2014;8(7):1208-19.

34. Fang $Y$, et al. Transcriptome sequencing reveals key pathways and genes associated with cisplatin resistance in lung adenocarcinoma A549 cells. PLoS One. 2017:12(1):e0170609.

35. Saber A, et al. Genomic aberrations in crizotinib resistant lung adenocarcinoma samples identified by transcriptome sequencing. PLoS One. 2016;11(4):e0153065.

36. Lan D, et al. Transcriptome profile and unique genetic evolution of positively selected genes in yak lungs. Genetica. 2018;146(2):151-60.

37. Girardin SE, et al. CARD4/Nod1 mediates NF-kappaB and JNK activation by invasive Shigella flexneri. EMBO Rep. 2001;2(8):736-42.

38. Kobayashi K, et al. RICK/Rip2/CARDIAK mediates signalling for receptors of the innate and adaptive immune systems. Nature. 2002;416(6877):194-9.

39. Pauleau AL, Murray PJ. Role of nod 2 in the response of macrophages to toll-like receptor agonists. Mol Cell Biol. 2003;23(21):7531-9.

40. Martin SA, et al. Directional responses following recombinant cytokine stimulation of rainbow trout (Oncorhynchus mykiss) RTS-11 macrophage cells as revealed by transcriptome profiling. BMC Genomics. 2007:8(1):150.

41. Zou J, et al. Functional characterisation of the recombinant tumor necrosis factors in rainbow trout, Oncorhynchus mykiss. Dev Comp Immunol. 2003; 27(9):813-22.

42. Horscroft JA, et al. From the Cover: Metabolic basis to Sherpa altitude adaptation[J]. Proceedings of the National Academy of ences of the United States of America. 2017:6382. https://doi.org/10.1073/pnas.1700527114.

43. Je JH, et al. NF-KB activation mechanism of 4-hydroxyhexenal via NIK/IKK and p38 MAPK pathway. FEBS Lett. 2004;566(1-3):0-189.

\section{Publisher's Note}

Springer Nature remains neutral with regard to jurisdictional claims in published maps and institutional affiliations.

Ready to submit your research? Choose BMC and benefit from:

- fast, convenient online submission

- thorough peer review by experienced researchers in your field

- rapid publication on acceptance

- support for research data, including large and complex data types

- gold Open Access which fosters wider collaboration and increased citations

- maximum visibility for your research: over $100 \mathrm{M}$ website views per year

At BMC, research is always in progress.

Learn more biomedcentral.com/submission 